\title{
Efavirenz Interference in Urine Screening Immunoassays for Tetrahydrocannabinol
}

\author{
Nicholette M Oosthuizen ${ }^{1}$, Johannes B Laurens ${ }^{2}$ \\ ${ }^{1}$ Department of Chemical Pathology, University of Pretoria/National Health Laboratory \\ Service, Tshwane Academic Division \\ ${ }^{2}$ Department of Chemistry, University of Pretoria \\ Corresponding author:
}

Dr Nicky Oosthuizen, PO Box 2034, Department of Chemical Pathology, University of Pretoria, PRETORIA, South Africa, 0001

Tel: +27 123192114 Fax: +27 123283600

E-mail address: nicky.oosthuizen@up.ac.za

\section{DECLARATIONS}

Competing interests: None

Funding: This research was funded by the University of Pretoria

Ethical Approval: The Faculty of Health Sciences Research Ethics Committee of the University of Pretoria approved this study (S110/2009)

Guarantor: NMO

Contributorship: The study was conceived by JBL. NMO reviewed the literature and both authors contributed to protocol development. NMO was involved in gaining ethical approval and interpreted laboratory results. JBL supervised GC-MS analysis, performed deconjugation experiments and interpreted laboratory results. NMO wrote all drafts of the manuscripts. Both authors reviewed and edited the manuscript and approved the final version.

Acknowledgements: We are grateful to Bronwyn Adendorff for sample collection and processing, and collation of laboratory results. We thank Adriaan Marais for technical assistance with the GC-MS analysis. We thank Homemed Medical Diagnostics for donating THC urine test strips from ACON laboratories, and Roche Diagnostics and Ampath Laboratories for providing reagents and THC testing free of charge. 


\section{Abstract}

Background: It has been known for some time that the antiretroviral drug, Efavirenz (EFV), cross-reacts in urine immunoassays for tetrahydrocannabinol (THC). Because published studies investigating this phenomenon are limited, cross-reactivity information for several immunoassays is lacking. Reports of possible false positive THC results from clinicians conducting workplace testing prompted us to investigate crossreactivity for assays frequently employed in our own setting. In light of the potentially deleterious consequences of misclassification, information about EFV cross-reactivity should be included in product information to facilitate interpretation of results and assay selection.

Methods: Random urine samples from 30 patients on EFV therapy were analyzed for THC metabolites by two near-testing devices (ACON Laboratories and Rapid Response ${ }^{\circledR}$ Drugs of Abuse test strips) and two automated immunoassays (Roche Diagnostics Cannabinoids II and Beckman Coulter SYNCHRON® systems THC2). THC confirmatory testing was performed by gas-chromatography mass spectrometry (GC-MS).

Results: GC-MS failed to detect THC metabolites in any of the samples, as did three of the four immunoassays. However, the Rapid Response ${ }^{\circledR}$ test strips yielded positive results in 28 out of 30 samples, which could be reversed on retesting after sample pre-treatment with glucuronidase. Conclusion: Our study supports previous findings that interference is attributable to a glucuronidated EFV metabolite. We postulate that crossreactivity is influenced by the composition of immunogens used to elicit anti- 
THC antibodies. Since access to such information is restricted, contributions from scientists in the antibody industry may be enlightening.

\section{Introduction}

Therapy with Efavirenz (EFV) is known to yield false positive results for tetrahydrocannabinol (THC) by several urine screening immunoassays. This interference has been attributed to EFV 8-glucuronide (EFV-8-G), one of two major urinary metabolites. The other major urinary metabolite, 8-hydroxy-EFV, and the parent drug do not interfere. ${ }^{1}$ Given the widespread inclusion of EFV in first-line antiretroviral therapy, the use of THC assays that are subject to EFV interference for workplace testing, is problematic. Not only may employees face punitive measures based on false positive results, but some may feel obliged to disclose their HIV status in order to refute a positive drug test. The aim of our study was to investigate EFV cross-reactivity for immunoassays commonly employed in the workplace and diagnostic laboratories in South Africa. These included two point-of care tests: Rapid Response ${ }^{\circledR}$ Drugs of Abuse Test Strip (BTNX Inc., Ontario, Canada), and THC One Step Marijuana Test Strip (ACON Laboratories, San Diego, USA); and two automated immunoassays: COBAS Integra Cannabinoids II (Roche Diagnostics, Indianapolis, USA), and SYNCHRON® systems THC2 (Beckman Coulter, Fullerton, USA).

\section{Methods and results}

Ethical approval for the study was obtained from the institutional Research Ethics Committee. Random urine samples were collected from 30 adult 
patients attending the antiretroviral clinic at the Tshwane District Hospital in Pretoria. To increase the likelihood that urinary excretion of EFV metabolites would be adequate, only patients who had been taking $600 \mathrm{mg}$ EFV daily for at least 14 days were selected. Samples were analyzed for THC metabolites by the screening immunoassays and confirmed by gas-chromatography mass spectrometry (GC-MS). Using the Rapid Response ${ }^{\circledR}$ assay, 28 out of 30 samples yielded positive results. All results obtained by the other immunoassays were negative. THC metabolite concentrations by GC-MS were undetectable in all samples, confirming false positive results for the Rapid Response ${ }^{\circledR}$ assay. On re-analysis by the Rapid Response ${ }^{\circledR}$ assay after hydrolysis using $\beta$-glucuronidase, positive samples reverted to negative.

\section{Discussion}

The fact that deconjugation reverses false positive THC results supports the hypothesis that interference is due to a glucuronidated EFV metabolite. In the two samples that tested negative from the outset, EFV-8-G concentrations may have been too low to cross-react significantly. Assuming that interference is due to structural similarity between EFV and cannabinoids, the findings of this study raise two interesting questions: a) why are only some immunoassays affected, and b) why is the interference particular to the glucuronidated metabolite? The answers may lie with assay-specific characteristics of the anti-THC antibodies. Comparisons of package insert data from ten assays ${ }^{2}$ for which EFV cross-reactivity information was available, (four from this and six from a published study ${ }^{1}$ ), revealed that interference was independent of host species or whether antibodies were 
mono- or poly- clonal (Table 1). Another determinant of antibody sensitivity and specificity in THC immunoassays is the position of carrier protein linkage. Being low molecular weight compounds, cannabinoid haptens (typically 11nor- $\Delta^{9}$-THC-9-carboxylic acid), require linkage to carrier proteins to produce immunogens for antibody generation. ${ }^{3,4}$ Steric hindrance by carrier proteins results in the generation of antibodies directed towards exposed functional groups remote from the site of carrier protein linkage. Linkage to positions C1, C2-, or C5'- elicits antibodies with high selectivity for the cyclohexyl ring, which translates to lower sensitivity to C8- and C11- hydroxylated metabolites and their glucuronides (Figure 1). ${ }^{4}$ Conversely, linkage to the cyclohexyl ring generates antibodies with greater cross-reactivity to C8-, C9- and C11substituted moieties. ${ }^{3}$ This concept was taken one step further by Roche Diagnostic Systems with the development of a non-cannabinoid immunogen containing only the benzpyran core. ${ }^{4}$ By eliminating the antigenic determinants of the cyclohexyl ring completely, antibodies with two to three times higher cross-reactivity to C8- and C11- hydroxylated metabolites than even the C9- linked immunogens were elicited. ${ }^{4}$ Antibodies generated by C8to C11- linked and benzpyran immunogens therefore offer superior detection for clinical purposes, because THC is extensively metabolized at these positions prior to urinary excretion. ${ }^{3}$ Inspection of the cross-reactivity data (albeit incomplete) for the ten assays in Table 1 reveals that some assays, which are subject to EFV interference, exhibit comparatively lower sensitivities to C8- and C11- hydroxylated metabolites. Our postulate that antibodies used in these assays may have been elicited using immunogens with carrier protein linkage to C1-, C2-, or C5' could not be verified, because vendor and 
Table 1. Comparison of urine screening immunoassays for tetrahydrocannabinol metabolites

\begin{tabular}{|c|c|c|c|c|c|c|c|c|c|c|}
\hline Immunoassay & SYNCHRON THC2 & $\begin{array}{l}\text { COBAS Integra } \\
\text { Cannabinoids II }\end{array}$ & $\begin{array}{l}\text { AXSYM } \\
\text { Cannabinoids }\end{array}$ & $\begin{array}{l}\text { One Step } \\
\text { Marijuana }\end{array}$ & $\begin{array}{l}\text { Syva Emit } \\
\text { d.a.u. }\end{array}$ & $\begin{array}{l}\text { Rapid } \\
\text { Response }\end{array}$ & $\begin{array}{l}\text { CEDIA } \\
\text { THC }\end{array}$ & $\begin{array}{l}\text { THCA/CTHC } \\
\text { Direct ELISA }\end{array}$ & Triage TOX & $\begin{array}{l}\text { Instant-View } \\
\text { THC }\end{array}$ \\
\hline Supplier & Beckman Coulter & Roche & Abbott & ACON & Dade Behring & BTNX & Microgenics & Immunalysis & Biosite & Alfa Scientific \\
\hline Method principle & $\mathrm{EIA}^{a}$ & $\mathrm{MAI}^{b}$ & $\mathrm{FPIA}^{c}$ & $\mathrm{LFIC}^{d}$ & $\mathrm{EIA}^{a}$ & $\operatorname{LFIC}^{d}$ & CEDIA $^{e}$ & ELISA $^{f}$ & $\mathrm{FIA}^{g}$ & $\operatorname{LFIC}^{d}$ \\
\hline Anti-THC Antibodies & $\begin{array}{l}\text { Mouse } \\
\text { monoclonal }\end{array}$ & $\begin{array}{l}\text { Mouse } \\
\text { monoclonal }\end{array}$ & $\begin{array}{l}\text { Sheep } \\
\text { polyclonal }\end{array}$ & $\begin{array}{l}\text { Mouse } \\
\text { monoclonal }\end{array}$ & $\begin{array}{l}\text { Mouse } \\
\text { monoclonal }\end{array}$ & $\begin{array}{l}\text { Rabbit } \\
\text { monoclonal }\end{array}$ & $\begin{array}{l}? \\
\text { monoclonal }\end{array}$ & $\begin{array}{l}\text { Sheep } \\
\text { polyclonal }\end{array}$ & $\begin{array}{l}\text { Mouse } \\
\text { monoclonal }\end{array}$ & $\begin{array}{l}\text { Mouse } \\
?\end{array}$ \\
\hline EFV cross-reactivity & No & No & No & No & No & Yes & Yes & Yes & Yes & Yes \\
\hline \multicolumn{11}{|l|}{ Cross-reactivity data } \\
\hline Cut-off (ng/mL) & 20 & 50 & 13 & 50 & 50 & 50 & 50 & 50 & 50 & 50 \\
\hline 11-nor- $\Delta^{8}-\mathrm{THC}-9$ COOH & 30 & 89 & 25 & 30 & & 50 & 40 & 11 & 40 & 50 \\
\hline 11-nor- $\Delta^{9}-\mathrm{THC}-9 \mathrm{COOH}$ & 20 & 50 & & 50 & 50 & 50 & 50 & & 50 & 50 \\
\hline 11-nor- $\Delta^{9}$-THC-9 COOH-Glu & 50 & 62 & & & & & & & 100 & \\
\hline 11-OH- $\Delta^{9}-\mathrm{THC}$ & 50 & 115 & 25 & & 90 & $>100000$ & 125 & $>1000$ & 150 & 100 \\
\hline $8-\beta-O H-\Delta^{9}-\mathrm{THC}$ & 50 & & & & 65 & & 1000 & & & \\
\hline 8- $\beta-11-$ dihydroxy- $\Delta^{9}-$ THC & 30 & 58 & & & 50 & & 5000 & $>1000$ & & \\
\hline Cannabinol & 100 & 2031 & 80 & 20000 & & 20000 & 1000 & $>1000$ & 2000 & \\
\hline
\end{tabular}




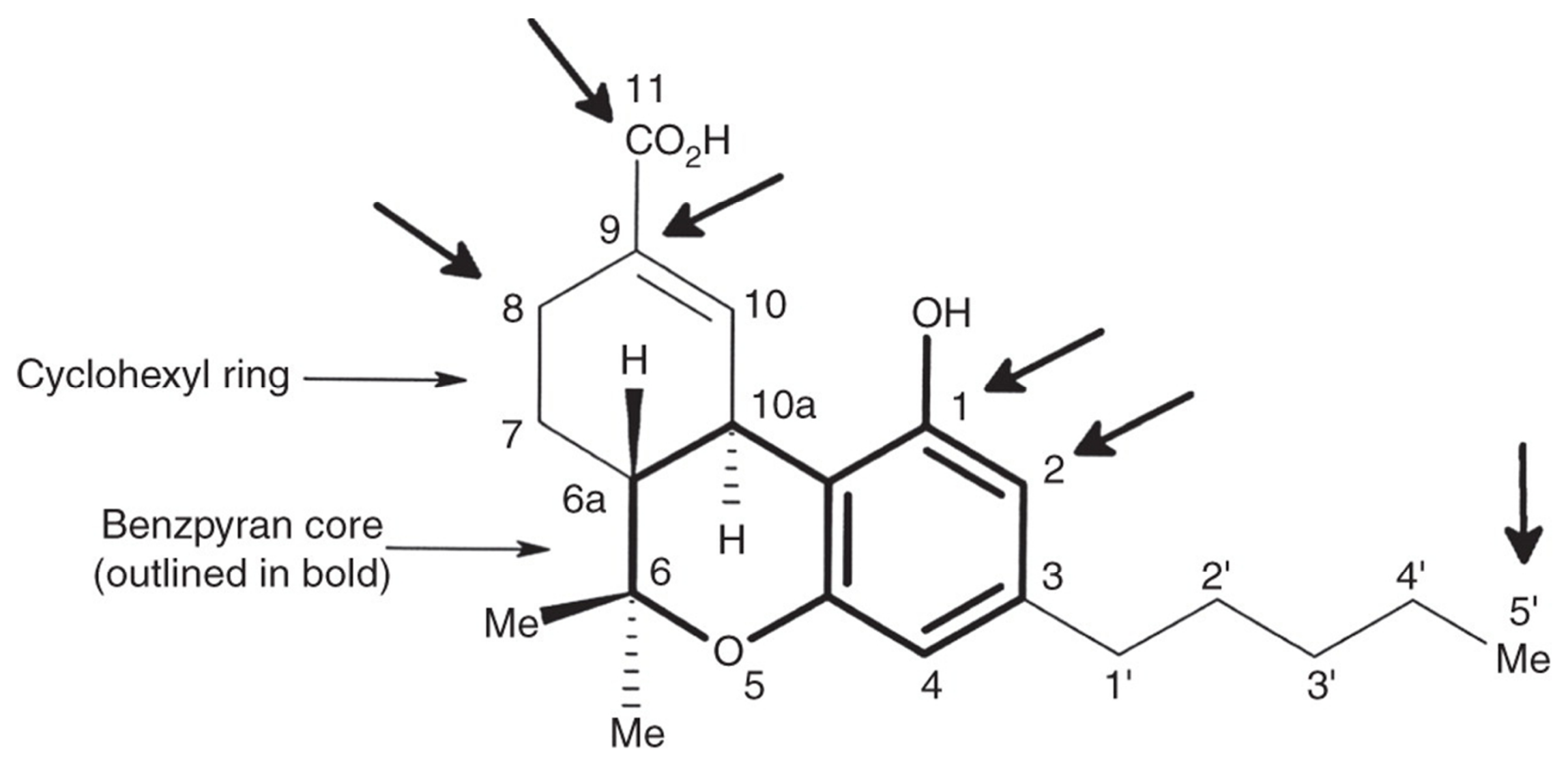

Figure 1. $\Delta^{9}$-11-nor-9-carboxytetrahydrocannabinol. Bold arrows indicate published linkage sites for carrier proteins to cannabinoid haptens (from reference 3). 
proprietary restrictions prevent access to detailed information about immunogen composition.

\section{Conclusion}

Further elucidation of the mechanisms of EFV interference may require inputs from the antibody industry or the field of molecular modelling. Interestingly, media reports have in recent years described the emergence of EFV abuse in South Africa, whereby crushed tablets are smoked for their psychoactive effects. ${ }^{5}$ Whether these effects are mediated by interaction of EFV or its metabolites with the endocannabinoid system is a question for future research into the psychopharmacology of EFV.

\section{References}

1. Rossi S, Yaksh T, Bentley H, et al. Characterization of interference with 6 commercial $\Delta^{9}$-tetrahydrocannabinol immunoassays by Efavirenz (glucuronide) in urine [Letter]. Clin Chem 2006;52:896-7.

2. Immunoassay package inserts: BTNX Inc. Rapid Response ${ }^{\circledR}$ Drugs of Abuse Test Strip; ACON Laboratories THC One Step Marijuana Test Strip; Roche Diagnostics COBAS Integra 400/700/800 Cannabinoids II; Beckman Coulter SYNCHRON® systems THC2; Microgenics Corp. CEDIA ${ }^{\circledR}$ Multi Level THC Assay; Immunalysis Corp. Cannabinoids (THCA/CTHC) Direct ELISA Kit; Dade Behring Inc. Syva ${ }^{\circledR}$ Emit ${ }^{\circledR}$ d.a.u. ${ }^{\circledR}$ Cannabinoid 50 ng Assay; Abbott Laboratories AXSYM System Cannabinoids; Biosite Inc. Triage TOX Drug Screen; Alfa 
Scientific Designs Inc. Instant-View® Marijuana (THC) Urine Cassette Test.

3. Tsai JS. Immunoassays for the detection of cannabis abuse. In:

EISohly MA, ed. Marijuana and the cannabinoids. $1^{\text {st }}$ Ed. Totowa: Humana Press, 2007:145-178.

4. Salamone SJ, Bender E, Hui RA, et al. A non-cannabinoid immunogen used to elicit antibodies with broad cross-reactivity to cannabinoid metabolites. J Forensic Sci 1998;43:821-6.

5. http://www.cnsproductions.com/drugeducationblog/in-the-news/447/ (Accessed September 2010). 